One of SIRA's many projects is making thin film microcircuits by laser micromachining. A magnified pattern of the film to be removed is reproduced in a mask which is illuminated by a $Q$-switched pulse of radiation from a laser. The optical image of the mask is focused on to the thin film and, when the laser is fired, the areas of the film exposed to the laser radiation evaporate. Successful experiments have been carried out with nichrome, cermet, chromium, gold and aluminium. The possibility of applying this technique to graticule production is also being studied.

\section{Elephants Caught}

AN aerial technique employing poisoned darts, shot from a crossbow by a marksman in a helicopter, has been used to capture young elephants during preliminary trials in the culling of herds in the Kruger National Park. Dr U. de V. Pienaar describes in the latest edition of the Journal for Scientific Research in the National Parks of the Republic of South Africa (No. $101 ; 1967)$ how a method already used successfully to catch rhinoceros in Kenya has been applied to young elephants-poisoned darts are already used to capture adults from the ground.

A helicopter first located a herd of breeding elephants, and waited until the ground party arrived. The helicopter then swooped in on the herd, usually causing it to stampede, and at a range of twenty-five to thirty yards suitable calves were shot with darts containing $1 \mathrm{mg}$ of propylorvinol hydrochloride, and $7 \mathrm{mg}$ of acetylpromazine maleate in a volume of $3 \mathrm{ml}$. After about 10-15 min the calves which had been hit fell to the ground, and the ground party moved in. A nylon thread was passed around the elephant's neck and this was pulled into an open crate. The antidote to the immobilizing mixture was administered to the earvein and the animal rose to its feet within minutes to be guided into the crate. Any mothers which attempted to rescue their young, which they often did, were kept away by the helicopter. In cases where the mother refused to leave a very young calf she was also immobilized.

After being tamed and adjusted to captivity the young elephants were shipped to America. The method proved very successful; twenty-seven young elephants, aged between 12 and 56 months, were captured in seven days of hunting. The great danger involved when breeding herds are disturbed by hunters operating on foot is avoided with the use of the helicopter, and the captures are made without damage to elephants or hunters.

\section{Ångstrom Ruler}

\section{from our Molecular Biology Correspondent}

THE phenomenon of excitation energy transfer has found occasional use in biochemistry, notably as a method for following binding processes by sensitization or quenching of fluorescence. Despite much interest and effort, however, its application to the study of topological detail in macromolecules has for the most part given trivial and disappointing results. Promise of better things comes from work of Stryer and Haugland (Proc. Nat. Acad. Sci. US, 58, 719; 1967), which should both please the spectroscopist and stimulate the biochemist.
An energy transfer system is set up when two fluorescent molecules are brought into proximity to one another; if there is large overlap between the emission spectrum of the first and the absorption spectrum of the second, and the geometry is favourable, excitation of the first molecule (the sensitizer) by irradiation at a wavelength in its absorption band leads to fluorescence from the second (the emitter). In such a system, no matter what the wavelength of excitation, fluorescence will always come from the species emitting at the lowest energy (longest wavelength). The theory of energy transfer, in terms of resonance between the transition dipoles of the two molecules, was developed twenty years ago by Förster, and predicts that the extent of transfer will vary inversely as the sixth power of the mean distance between the molecules $\left(r^{-6}\right.$. dependence). Whatever the situation in free solution there seems little doubt that excitation transfer between chromophores fixed in a macromolecule must proceed by this mechanism. Stryer and Haugland use the $\alpha$-naphthyl group as sensitizer and the dansyl group as emitter. These are attached at opposite ends of an oligoproline chain. A series of such sandwich molecules has been prepared, in which the length of the proline chain varies from one up to twelve residues. The advantage in using prolines as spacers is that at chain lengths greater than five a rigid helical structure (polyproline II helix) is formed, so that the separation of the two chromophores is always more or less precisely fixed. Further, the complete depolarization of the dansyl fluorescence in these compounds, when observed in a rigid glass matrix, shows that the chromophore is freely rotating; consequently there are no complications from differences in the relative orientation of the chromophores (since the efficiency of transfer depends also on the angle between the two transition dipoles).

The extent of energy transfer is determined from the excitation spectrum: the intensity of fluorescence from the dansyl group is measured with varying excitation wavelength. If there is no energy transfer, the excitation profile simply follows the absorption spectrum of the dansyl group, whereas for complete transfer it follows in effect the sum of the absorption spectra of sensitizer and emitter.

From the crystallographic dimensions of the polyproline II helix one can determine the separation of the two chromophores for each compound in the series, and it is found that the extent of energy transfer falls from 100 per cent when one proline residue separates the chromophores to 16 per cent when therc are twelve prolines; moreover, it is found that the $r^{-6}$-law holds with remarkable precision (an exponent of 5.9 is found). This is a pleasing result, for it provides the most conclusive evidence yet in favour of the Förster theory.

Stryer and Haugland refer to these measurements of energy transfer as a function of distance as a spectroscopic ruler, and indeed the method holds out the promise of applications to biological molecules. There are many systems in which there is a possibility of attaching a sensitizer and an emitter at functional sites on a macromolecule or assemblage of molecules so as to determine their separation. Whether such an elegant approach is in practice too good to be trueat least as far as more complex systems are concerned -will remain to be seen, but there is no shortage of applications that come to mind. 